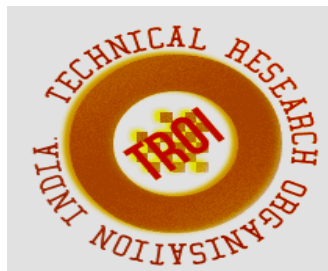

\title{
SMART GREEN HOUSE MONITORING AND CONTROLLING SYSTEM USING IOT
}

\author{
Rakesh B N ${ }^{1}$, Navasanthanalakshmi $\mathrm{A}^{2}$, Savitha $\mathrm{C}^{3}$, Varalakshmi $\mathrm{V}^{4}$, Thejeshwini $\mathrm{H}^{5}$ \\ ${ }^{1}$ Assistant professor, ${ }^{2,3,4,5}$ Student \\ Department of Electronics and Communication \\ Dr.T.Thimmaiah Institute of Technology \\ 1rakesh.bn07@gmail.com, ªnslnava90@gmail.com
}

\section{ABSTRACT}

The greenhouse industry is the fastest growing sector worldwide. The smart greenhouse separates the crop from the environment, thus the greenhouse providing some way of shelter from the direct sunlight of the external weather conditions. This system propose a contribution to the development of smart greenhouse monitoring which presents the design and development of an electronic system based on a 32-bit ARM microcontroller that integrates remote sensing functions rooted in the cloud computing using Internet of Things (IoT). This system acquires the data from soil moisture sensor, humidity sensor, temperature sensor, and light sensor $24 \times 7$, if the soil moisture is found to be low it turns on the water pump to sprinkle water until the soil is moist, if the temperature in the green house is found to be high a fan is turned on , if temperature is low room heater is switched On , similarly if the light intensity is low LED lamp is turned ON. If the humidity is low then a humidifier is turned on .In addition to this the system also sends all four sensor values over internet for remote monitoring and control of LED lamp, room heater, humidifier, or fan can be done manually if needed through internet . we have also proposed live video streaming on internet with the help of Raspberry pi 3 and camera. Keywords: greenhouse, microcontroller, Internet of Things (IoT), Raspberry pi 3.

\section{INTRODUCTION}

The smart greenhouse industry is the fastest growing sector worldwide. This smart greenhouse separates the crop from the environment, thus the greenhouse system providing some way of shelter from the direct sunlight of the external weather conditions. By using this method it enables us in the production of crops which can be produced to a specific location. The smart greenhouse helps us in the manipulation of the crop environment. By allowing this method the farmer can improve the cultivation in such a way that the plants need. By using this method it leads to higher crop yield, prolonged production period, better quality, and less use of protective chemicals. In smart greenhouse the crops added value per unit area like fertilizers, urea etc., is much higher than that in open-field cultivation. By looking in climate condition, the energy is required more in moderate climate zones, whereas in grid zones, the cooling and availability of water, temperature, light is of major concern.

The use of materials and energy as well as crop yield and quality can be controlled by operating the adjustable components of greenhouse, such as heating and cooling inputs, window opening, drip irrigation, screening and CO2 dosage. Hence, it can be expected that the way these controls are operated by using a 32bit ARM microcontroller and the final economic result. If the user is in abroad the conditions of the crops and resource management in smart greenhouse is difficult to know the control variables with a remote sensing system using the IoT. This is because it is almost difficult for the farmerto manipulate and be present every day near the system. Indeed, remote communication systems are a 
major component of the policy of modernization and technology transfer, due to the increasing development of mobile telecommunications.

Internet of Things (IoT) is the network of physical things embedded with electronic circuits, sensors, software and network connection which enables these things to exchange data from one another. The IoT was started in the year 1998 and the term Internet of Things was first coined by Kevin Ashton in 1999. IoT is the fusion of the digital and physical world. In a world of IoT, millions of things or devices will be interconnected and uniquely identified on the Internet. The Internet of Things allows objects to be sensed and controlled remotely across existing network infrastructure, creating opportunities for more direct integration between the physical world and computer-based systems, and resulting in improved efficiency, accuracy and economic benefit. In near future, IoT is expected to provide many more services like advanced connectivity of physical objects over a wide network and also many applications.

The system proposes a contribution to the development of greenhouse monitoring. This proposed project presents the design and development of an electronic system based on a microcontroller that integrates remote sensing functions rooted in the cloud computing using Internet of Things (IoT).

The proposed system acquires the data from soil moisture sensor, humidity sensor, temperature sensor, and light sensor $24 \times 7$, if the soil moisture is found to be low it turns on the water pump to sprinkle water until the soil is moist, if the temperature in the green house is found to be high a fan is turned on, if temperature is low room heater is switched on, similarly if the light intensity is low LED lamp is turned ON. If the humidity is low then a humidifier is turned on, In addition to this the system also sends all four sensor values over internet for remote monitoring and control of LED lamp , room heater, humidifier , or fan can be done manually if needed through internet . We have also proposed live video streaming on internet with the help of Raspberry pi 3 and camera.

\section{LITERATURE REVIEW}

K.Rangan et al., [1] proposed a system based on the measuring of parameters like Water $\mathrm{pH}$, Soil wetness, Light intensity, Humidity and temperature, these parameters are obtained by using the sensors ,the data from the each sensors where measured, processed, controlled and updated to owner through SMS using GPS modem. The output from the sensors is continuously given to the PIC microcontroller the parameters like temperature, $\mathrm{pH}$, humidity, soil wetness and light intensity are varied from set parameters, which is continuously monitored and updated in the mobile receiver. As GSM is used, it is possible only to monitor the system but cannot control it and PIC microcontroller requires external circuits to be connected which increases the cost of the system.

Prachi Patil et al., [2] proposed the Real time automation of agricultural environment, using PIC16F877A and GSM SIM300 modem is focused on automating the irrigation system for social welfare of Indian agricultural system. This system will be useful and helpful for the farmers as they can monitor the soil moisture condition of the farm as well as controls the soil moisture by monitoring the level of water in the water source and accordingly switches the motor ON/OFF for irrigation purposes. The circuit also monitors the water in the water source so that if the water level. The system also consists of a GSM modem through which the farmer can easily be notified about the critical conditions occurring during irrigation process. It requires bread board implementation.

P. S. Asolkar et al., [3] proposed a system which demonstrates about the effective method for crop monitoring in agriculture, which shows the path to rural farming community to replace traditional crop cultivation techniques. The main advantage is the use of GSM based control system which allows to control from a remote area to the desired location. The power consumed by control devices is very low which makes total annual cost also low. In addition, the expense required for controlling devices like fan and heater is calculated by farmers for crop taken in next season. But it cannot automate multiple green houses simultaneously.

Ravi Kishore Kodali et al., [4] proposed a model which helps the farmers to carry out 
the work in a farm automatically without the use of manual inspection. The irrigation of agriculture field is carried out using automatic drip irrigation. Proper water management tanks are constructed and they are filled with water after measuring the current water level using an ultrasonic sensor. Temperature and air humidity are controlled by humidity and temperature sensors. A tube well is controlled using GSM module. Further the readings collected from storage containers are uploaded to cloud service and can be forwarded to an ecommerce company. It reduces effort and time of farmer and makes farming efficient and profitable activity.

Arul Jai Singh et al., [5] explained about Green house monitoring system using PIC Microcontroller. The controller interacts with the various sensor modules like temperature sensor, humidity sensor, soil sensor and light sensor in real-time in order to control the light, aeration and drainage process efficiently inside greenhouse by actuating a dripper, cooler, fogger and lights according to the necessary condition of the crop growth. Their advantage is providing maximum automation, labour saving, natural resource like water saved to a great extent etc. The response of the system is to measure and control the four parameters for growth of the plant in the greenhouse, i.e., temperature, humidity, soil moisture, and light intensity

KasimM AI-Aubidy et al., [6] briefed about the design and implementation of $\mathrm{A}$ real time monitoring and control of several environmental parameters for group of green houses based on wireless sensor network. The main objective of the proposed project is to monitor and control the climatic condition inside each greenhouse according to the desired values of crop. The farmer can remotely select a greenhouse to monitor the measured variables and turn ON or OFF selected actuators using either his smart phone or internet access. Live video is available through adjustable web camera to monitor crop growth and health. The farmer can send commands to turn ON or OFF certain devices in a selected greenhouse through wireless communications.

\section{METHODOLOGY}

The basic idea of this project is to fully automate the green house and store the data in the cloud. This can be achieved by the use of IoT linked cloud services applications.

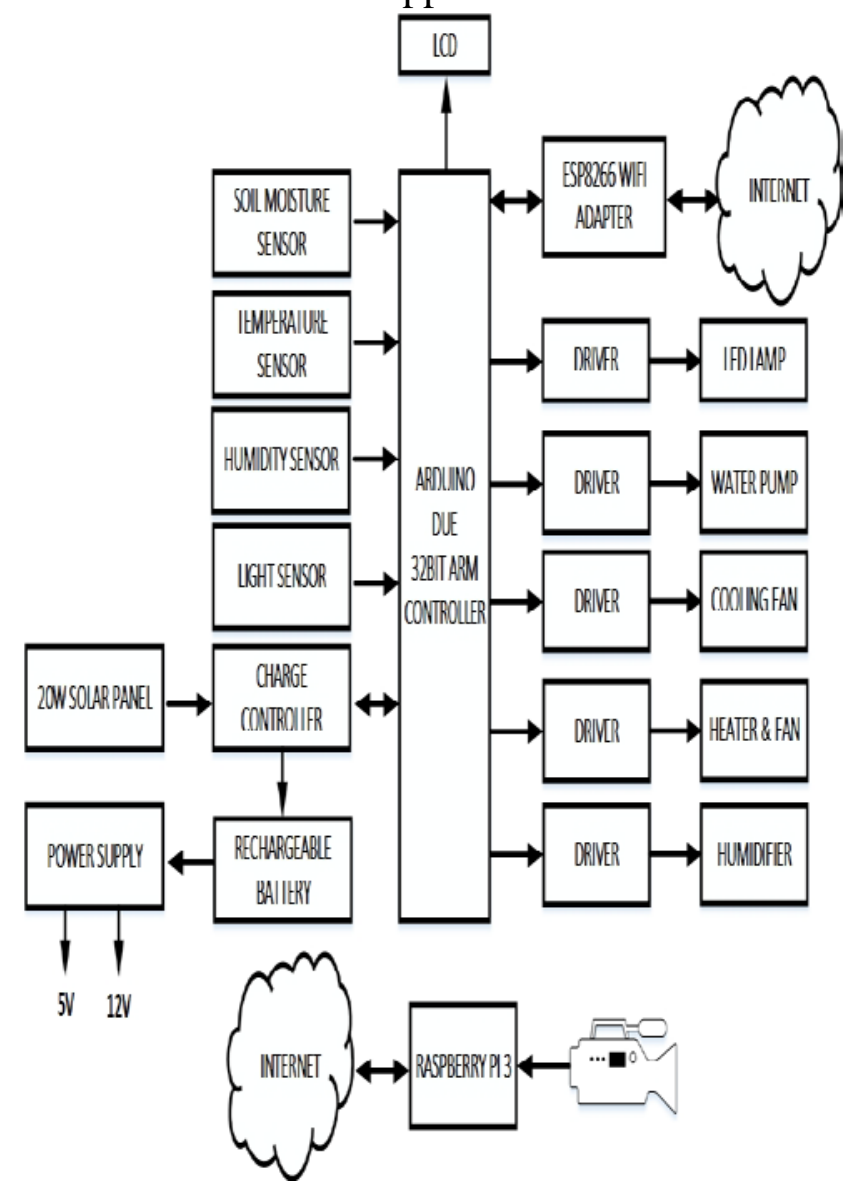

\section{Figure 1: Block diagram of Smart Green house}

At first each sensor values are connected to the Arduino board and the values are stored in the cloud and it can be accessed and controlled using the Blynk app. Many such sensors are linked to an Arduino board which can oversee the working of each and every sensor. It provides a live video streaming which is very useful for the user to monitor the green house sitting from anywhere in the world through Blynk app.

\section{Arduino Due}

The Arduino Due (32-bit microprocessor) is setup near the green house that consists of sensors on the either side. These sensors are useful to get the real time data. Every sensor values are sent to the Arduino board and these values are processed and the appropriate parameter is monitored as well as controlled .The Arduino Due is a microcontroller development board based on the Atmel SAM3X8E ARM Cortex-M3 CPU - 
AT91SAM3X8E. It is a 32-bit Arm core microcontroller development board.

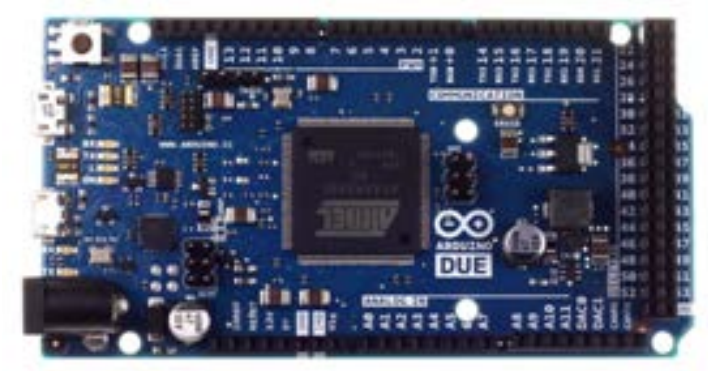

Figure 2: Arduino Due

Arduino Due has 54 digital input/output pins 12 of which are used as PWM outputs. It consists of 12 analog inputs, 4 UARTs (hardware serial ports).It operates at a clock speed of $84 \mathrm{MHz}$, an USB OTG capable connection, 2 TWI, 2 DAC (digital to analog), a power jack, an JTAG header, a SPI header, a reset button and an erase button. The board should be connected to the computer using a micro-USB cable or it can be powered up with an AC-DC adapter or with a battery to get started and it contains everything needed to support the microcontroller.

\section{ESP8266}

The ESP8266 is a low-cost Wi-Fi microchip delivers highly Integrated Wi-Fi SoC. It is a transceiver where the data can be sent and received. This module is used for WiFi connectivity from here the Blynk app can access the data.

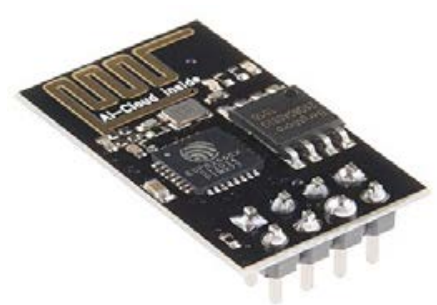

Figure 3: ESP8266

\section{Temperature and Humidity Sensor (DHT22)}

DHT22 use the humidity sensing component which has two electrodes with moisture holding substrate between them so as the humidity changes, the conductivity of the substrate changes or the resistance between these electrodes changes.

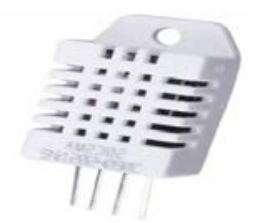

Figure 4: DHT22

For measuring temperature these sensors use a NTC temperature sensor or a thermistor. DHT22 sensor requires 3 to $5 \mathrm{~V}$ power and $2.5 \mathrm{~m}$. A max current. As the name suggests, these are used for monitoring the temperature and used to check whether the temperature is high or low inside the green house. And a critical value of temperature is fixed. And when sometimes the temperature inside the greenhouse reaches above the critical temperature level, the coolant is provided to cool down the temperature and bring it down to the favorable required temperature inside the green house.

\section{Soil Moisture sensor}

Soil moisture sensors measure the volumetric water content in soil. Resistivity of a Soil is a measuring of how strongly the soil will resists the flow of electricity between two electrodes that can be used to determine the moisture content of the soil.The volumetric content of the water is measured using the two probes of a soil moisture sensor. The two probes allow the current to pass through the soil and then it gets the resistance value to measure the moisture value.

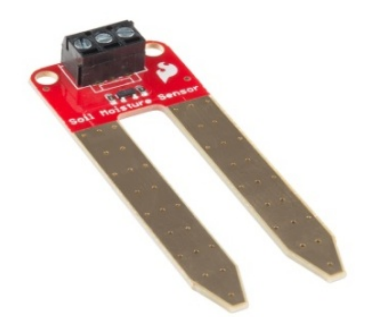

\section{Figure 5: Soil moisture Sensor}

\section{Light-Dependant Resistor (LDR)}

LDR (Light Dependent Resistor) is the light sensor which is usually used for the purpose. Its main function is to monitor the intensity of light. When light are required in the greenhouse then the lights are turned on and when it needs to save the power, it turns off the light. 


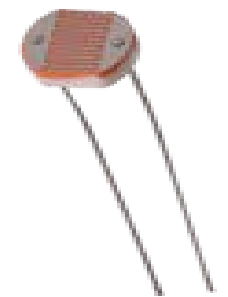

Figure 6: $L D R$

A LDR is made of a high resistance semiconductor. LDR is also called as a photo resistor or photocell is a resistor which exhibits photoconductivity whose resistance decreases with increasing incident light intensity. The photons are absorbed by the semiconductor if light falling on the device is of high enough frequency, which give bound electrons enough energy to jump into the conduction band. The resulting free electron (and its hole partner) conduct electricity, thereby lowering resistance.

\section{Liquid Crystal Display (LCD)}

A Liquid Crystal Display (LCD) is a low cost, low power device capable of displaying text and images. LCDs are used to display the sensor data like the green house humidity, temperature, soil moisture and light intensity. The LCD is the user interface where he can know the present condition of the Green house. Based on the amount of data to be displayed, this project uses a 20X4 LCD.

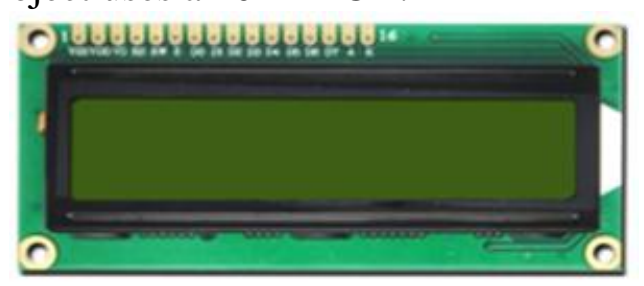

Figure 7: LCD

This project uses a 20X4 LCD display which requires an operating voltage of $5 \mathrm{~V}$ with 16 pins.

\section{Relay}

Relay is simply an electromagnetic switch which helps in opening and closing of circuits electronically. It has four important components-electromagnet, an armature, a spring and set of electrical contacts.

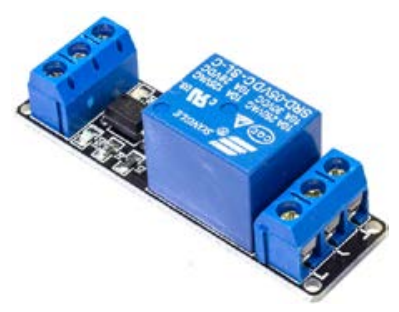

Figure 8: Relay

In short it is a magnetism device for remote or automatic management that's motivated by variation in conditions of an electrical circuit which operates successively alternative devices (such as switches) within the same or a special circuit. The sensors give the accurate value or status of temperature, humidity, light and smoke inside the green house.

\section{Raspberry Pi}

The Raspberry $\mathrm{Pi}$ is a credit card sized single-board computer that is capable of many of the things that our desktop PC does, like spreadsheets, word-processing and games. The high definition video, as well as still photographs are captured by the Camera Module. Through this camera module the user can view the green house from anywhere in the part of the world. So he can get the live video streaming of the green house through $\mathrm{Wi}-\mathrm{Fi}$ connectivity.

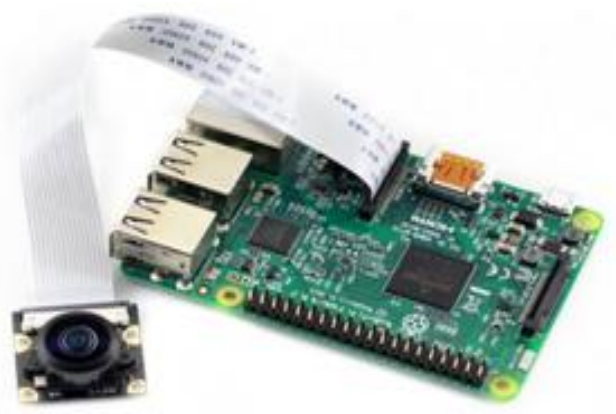

Figure 9: Raspberry Pi with Camera

\section{Solar Panel}

Photovoltaic module or photovoltaic panel is a packed interconnected assembly of photovoltaic cells, also known as solar cells. The photovoltaic module, known more commonly as the solar panel.

Solar panels use light energy (photons) from the sun to generate electricity through the photovoltaic effect (this is the photo- electric effect). The majority of modules use waferbased crystalline silicon cells or a thin-film cell based on cadmium telluride or silicon. 
Crystalline silicon, which is commonly used in the wafer form in photovoltaic (PV) modules.

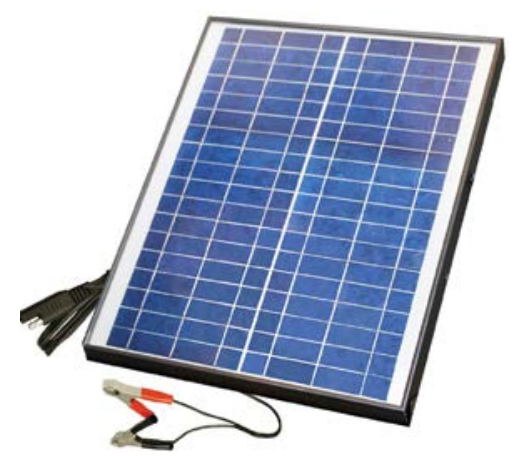

Figure 10: Solar Panel

The solar energy is a convectional energy which is available to us 24X7. The power needed for the sensors, LCD, Arduino board, water pump can be driven with the help of solar panels.

\section{IMPLEMENTATION}

Smart Green house monitoring and controlling is achieved with the help a remote sensing system called as Internet of Things (IoT) with sensors like soil moisture sensor which consists of two electrodes, a Humidifier, Temperature sensor, Light intensity sensor which are connected to 32 bit ARM processor. Get the data from the soil sensor LDR and Raspberry pi camera. The Arduino Due processes the data of this sensors and the status of these sensors are displayed using LCD. After displaying the humidity, temperature and soil moisture on LCD the data also send to Blynk web server where the sensor data are stored and can be retrieved by the user whenever the use needs and streams live video with the help of Raspberry Pi camera module.

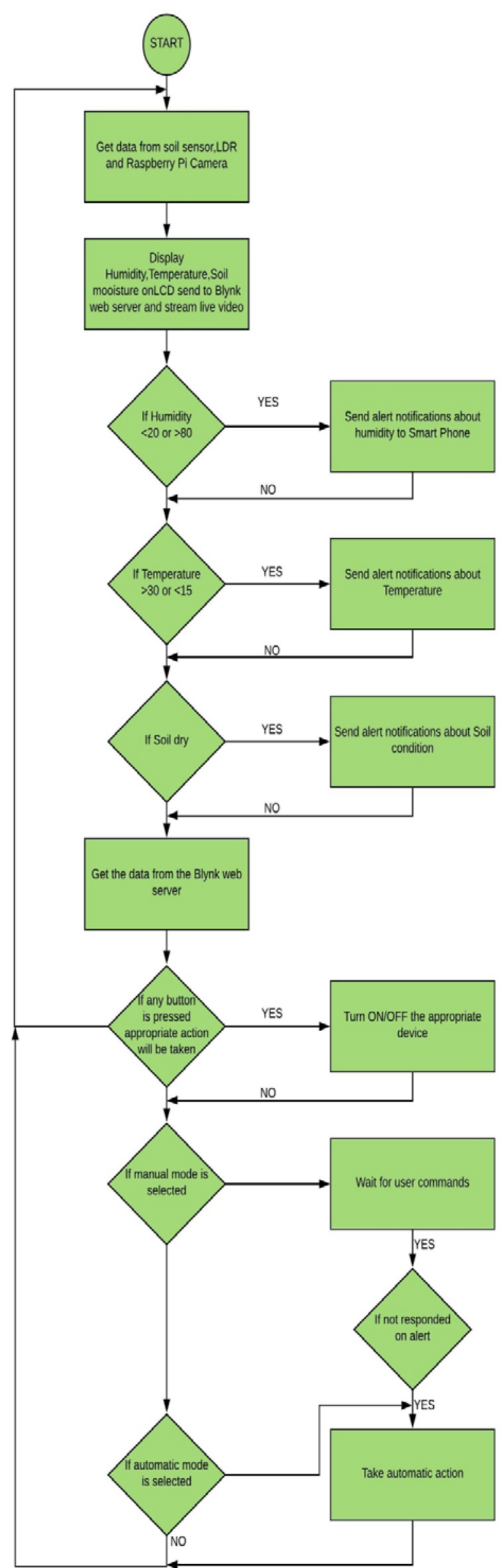

Figure 11: Flow Chart 
Initially all the sensor values like humidity, temperature, soil moisture, light intensity are obtained by the controller and are processed to get the accurate values by Arduino Due. Once the values are obtained it is displayed on the LCD for user interface to view the present condition of the green house so that the user can control the parameters of the green house accordingly. The parameters can be controlled using the Blynk app .Blynk app provides a graphical interface the control the green house free of cost. It provides user convenience and it can be designed according to the user.

Initially the controller will obtain all the sensor data and are processed to get the accurate values by Arduino Due .It will compare each parameter with the pre-initialized value and take action accordingly The system can operate in two modes either in manual mode or in automatic mode.

If humidity is less than or greater than the normal preset value it sends the alert notification about the humidity to Smart phone. If the temperature is less then or greater than the required value then it sends the alert notification to the user. Similarly it checks the next conditions; if the soil is dry it sends the alert notification about the soil dry to smart phone. The user will get data from the Blynk web server through the Blynk App using the Smart Phone. The user can monitor as well as can control using the Blynk App, buttons to turn on or off the devices will be created in the Smart phone using Blynk App. If any button is pressed appropriate action will be taken and then turns ON/OFF the appropriate

device, if no button is pressed the system checks whether the user has put in manual mode or automatic mode.

If manual mode is selected then wait for the user commands incase if the user selects in manual mode but he is not responding to the alert messages then the system takes automatic action. If automatic mode is selected then the system is ready to take an automatic action. After it goes to start again it checks each conditions and the process continuously works.

\section{V.APPLICATIONS}

i. Greenhouse technology is making it easier in order to study the potential value of medicinal plants and different ways to increase the plant yields and growth. It also makes plants more disease resistant.

ii. The purpose of the greenhouse is to shield crops from excess cold or heat and it also protects the crop from the unwanted pests. A greenhouse makes it possible to grow certain types of crops year round, and fruits, vegetables, and flowers.

iii. Greenhouses allows the farmers to monitor and control the conditions of their crops growing. The rate of photosynthesis can be increased using artificial heating lighting.

iv. The system is highly customizable and features low operating costs, easy operation, and remote control capability

v. Green House is environmental friendly and reduces pollution.

vi. Solar energy is used as a power supply in order to reduce the power consumption for the entire system.

vii. Data from soil moisture, temperature, humidity and light sensors are uploaded to the global cloud where the farmer can get the data from anywhere in the world at anytime.

viii. Real time monitoring and controlling of crops in green house is done through IoT.

ix. Live video streaming for monitoring the various requirements of the cropsis done using a raspberry pi and camera were the farmer can monitor the live growth and conditions of the crops.

\section{CONCLUSION}

The proposed system is going to play an important role in future of agriculture system and hopefully it would going to help in boosting the efficiency of growth and production of agriculture industry. The advantage of Smart Greenhouse over conventional farming is that we were able to produce insecticide and pesticide free crops and create a climate for the proper growth of plants and even provides alternative source of income through apiculture, selling tube well water etc. Moreover, this system can be installed by any individual in his house (Rooftop greenhouse), who does not have knowledge about farming. Since one can maintain any climatic condition in this type of Greenhouse, it is possible to cultivate any type of crop. Hence, we grow plants like Hibiscus, which are imported to India. We can reduce $70 \%-80 \%$ water requirement. It also increases 
yield and rate of growth and produces organic agricultural products. Most importantly, we are able to connect farmer directly to consumer using IoT, which can save him from the clutches of middle men. It reduces effort and time of farmer and makes farming efficient and profitable activity.

This project mainly focuses on the smart agriculture monitoring system using solar panel, which serves as a reliable and efficient system, and corrective action can be taken. Wireless monitoring of field reduces the human power and it allows user to see accurate changes in crop yield. It is cheaper in cost and consumes less power. This system is more efficient and beneficial for farmers. The green house works logically and performed activities based on various important parameters such as temperature, humidity and light as these three parameters are the most important parameters for the growth of plant and greenhouse monitors these parameters efficiently with live video streaming thus leading to better results as compared to traditional approach. Thus, this system seems to be more promising and can lead to better crop efficiency and better productivity. It would even send an alert message regarding the parameters such as air, humidity, temperature, soil, moisture through a pop-up message using mobile android applications. As solar energy source is a renewable energy source, it reduces electric bills, and has low maintenance cost.

\section{REFERENCES}

[1] K. Rangan, Indian Institute of Technology Madras, Chennai, India-An Embedded Systems Approach to Monitor Green House2010

[2] Prachi Patil, AkshayNarkhede, AjitaChalke, Harshali Kalaskar, Manita Rajput-RealTime Automation of AgriculturalEnvironment2014

[3] P. S. Asolkar E\&TC Department SSBT's COET, Bambhori Jalgaon, India- An Effective Method of Controlling the Greenhouse and Crop Monitoring Using GSM-2015

[4] Ravi Kishore Kodali, Vishal Jain and Sumit Karagwal- IoT based Smart Greenhouse2016

[5] Prathibha S R1, Anupama Hongal 2, Jyothi
M P3-IOT Based Monitoring System In Smart Agriculture-2017

[6] Zaidon Faisal Shenan- IOT Based Intelligent Greenhouse Monitoring and Controlling-2017

[7] Arul Jai Singh.S, Raviram.P, Shanthosh Kumar.K-Embedded based green house monitoring system using pic microcontroller2014

[8] Nilimamayee Samal and Umesh Chandra Pati- multi-channel data acquisition, data logging and supervisory control of different parameters for green house application-2015

[9] Kasim M. AI-Aubidy, Mohammad M. Ali, Ahmad M. Derbas \& Abdallah W. AIMutairi Real-Time Monitoring and Intelligent Control for Greenhouses Based on Wireless Sensor Network-2014

[10] AbhinavValada David Kohanbash George Kantor- Design and Development of aWireless Sensor Network System for Precision Agriculture-2010 